\title{
Superinfection Exclusion by p28 of Turnip Crinkle Virus Is Separable from Its Replication Function
}

\author{
Qin Guo, ${ }^{1}$ Shaoyan Zhang, ${ }^{1}$ Rong Sun, ${ }^{1}$ Xiaolong Yao, ${ }^{1}$ Xiao-Feng Zhang, ${ }^{1,2}$ Satyanarayana Tatineni, ${ }^{3}$ \\ Tea Meulia, ${ }^{1,4}$ and Feng $Q \mathbf{u}^{1, \dagger}$ \\ ${ }^{1}$ Department of Plant Pathology, Ohio Agricultural Research and Development Center, The Ohio State University, Wooster, $\mathrm{OH}$ \\ 44691, U.S.A. \\ ${ }^{2}$ Fujian Key Laboratory of Plant Virology, Institute of Plant Virology, Fujian Agriculture and Forestry University, Fuzhou, China \\ ${ }^{3}$ United States Department of Agriculture-Agricultural Research Service and Department of Plant Pathology, University of \\ Nebraska-Lincoln, Lincoln, NE 68583, U.S.A. \\ ${ }^{4}$ Molecular and Cellular Imaging Center, Ohio Agricultural Research and Development Center, The Ohio State University
}

Accepted 8 November 2019.

\begin{abstract}
We recently reported that the $\mathbf{p 2 8}$ auxiliary replication protein encoded by turnip crinkle virus (TCV) is also responsible for eliciting superinfection exclusion (SIE) against superinfecting TCV. However, it remains unresolved whether the replication function of p28 could be separated from its ability to elicit SIE. Here, we report the identification of two single amino acid mutations that decouple these two functions. Using an Agrobacterium infiltration-based delivery system, we transiently expressed a series of $\mathrm{p} 28$ deletion and point mutants, and tested their ability to elicit SIE against a cointroduced TCV replicon. We found that substituting alanine (A) for valine (V) and phenylalanine (F) at p28 positions 181 and 182, respectively, modestly compromised SIE in transiently expressed p28 derivatives. Upon incorporation into $\mathrm{TCV}$ replicons, V181A and F182A decoupled TCV replication and SIE diametrically. Although V181A impaired SIE without detectably compromising replication, $F 182 \mathrm{~A}$ abolished $\mathrm{TCV}$ replication but had no effect on SIE once the replication of the defective replicon was restored through complementation. Both mutations diminished accumulation of $\mathbf{p} 28$ protein, suggesting that p28 must reach a concentration threshold in order to elicit a strong SIE. Importantly, the severe reduction of $\mathrm{F} 182 \mathrm{~A}$ protein levels correlated with a dramatic loss in the number of intracellular p28 foci formed by p28-p28 interactions. Together, these findings not only decouple the replication and SIE functions of p28 but also unveil a concentration dependence for $p 28$ coalescence and SIE elicitation. These data further highlight the role of p28 multimerization in driving the exclusion of secondary TCV infections.
\end{abstract}

Keywords: translation and replication

${ }^{\dagger}$ Corresponding author: F. Qu; qu.28@osu.edu

Funding: This study was supported by a grant from the National Science Foundation (1758912); a SEEDS grant from the Ohio Agricultural Research and Development Center (OARDC); graduate assistantships from The Ohio State University and OARDC to Q. Guo and R. Sun, respectively; as well as tuition assistance to S. Zhang, R. Sun, and Q. Guo from the Department of Plant Pathology, The Ohio State University.

The author(s) declare no conflict of interest.

๑) 2020 The American Phytopathological Society
Many viruses block the subsequent entry or replication of the same or closely related viruses in the cells they occupy, through a process known as superinfection exclusion (SIE). These viruses include important human and animal pathogens such as human immunodeficiency virus, hepatitis $C$ virus, West Nile virus, and vesicular stomatitis virus (Nethe et al. 2005; Schaller et al. 2007; Simon et al. 1990; Tscherne et al. 2007; Zou et al. 2009), as well as plant pathogens such as tobacco mosaic virus, citrus tristeza virus, cucumber mosaic virus, wheat streak mosaic virus, turnip crinkle virus (TCV) (Bergua et al. 2014; Dietrich and Maiss 2003; Folimonova 2012; Julve et al. 2013; Miyashita and Kishino 2010; Takahashi et al. 2007), and, more recently, sonchus yellow net virus (Zhou et al. 2019). SIE in plant virus infections is also thought to underlie the welldocumented cross protection phenomenon (Folimonova 2013; Zhang and Qu 2016; Ziebell and Carr 2010). Cross protection insulates plants from a disease-causing virus by simply preinoculating them with a mild variant of the same virus. Although cross protection has been adopted for plant virus disease management for at least 50 years (Ziebell and Carr 2010), its underlying molecular mechanism remains poorly understood.

A number of earlier studies investigated SIE of plant viruses by tagging the same virus with two different fluorescent proteins (e.g., green fluorescent protein [GFP] and mCherry) (Dietrich and Maiss 2003; Julve et al. 2013; Miyashita and Kishino 2010; Takahashi et al. 2007; Tatineni and French 2016; Zhang et al. 2017). These studies demonstrated that most of the viruses excluded their own variants at the single cell level, thus implicating intracellular exclusion as one of the primary causes for SIE. Using this approach, Zhang and colleagues (2017) found that SIE in TCV infections occurred inside individual cells, and it acted to block the replication of the superinfecting TCV replicons. Strikingly, this replicational blockage was mediated by $\mathrm{p} 28$, one of the TCV-encoded proteins required for TCV genome replication (Zhang et al. 2017). The fact that $\mathrm{p} 28$, the auxiliary replication protein of TCV, functions to both facilitate and repress the replication of TCV genomic RNA (gRNA), along with the observation that fluorescent protein-tagged p28 formed large intracellular bodies that coalesced other p28 variants, prompted the hypothesis that p28 undergoes concentration-dependent conformational changes to accommodate its dual functions. Namely, the p28 protein assumes one conformation at lower concentrations to enable replication but another self-perpetuating conformation at higher concentrations to block replication (Zhang et al. 2017, 2018). 
TCV is a small icosahedral plant virus with a (+)-strand RNA genome that encodes five proteins. The $5^{\prime}$ proximal p28 and its C-terminally extended derivative (p88) are both translated directly from TCV gRNA, and are themselves required for gRNA replication (Fig. 1A). Because the production of $\mathrm{p} 88$ depends on infrequent translational read-through of the p28 stop codon (UAG), the intracellular concentration of p28 far exceeds that of p88 (White et al. 1995). However, p28 lacks the conserved RNA-dependent RNA polymerase (RdRP) motif and, hence, is known as the auxiliary replication protein. On the other hand, p88 is thought to be directly responsible for gRNA replication through its RdRP activity. TCV additionally encodes two small movement proteins, $\mathrm{p} 8$ and $\mathrm{p} 9$, that are translated from subgenomic RNA 1 (sgRNA1), and a capsid protein or silencing suppressor from sgRNA2 (Fig. 1A) (Cao et al. 2010; Qu et al. 2008; Zhang et al. 2012).

In the recent study by Zhang and colleagues (2017), we established that p28 was necessary and sufficient to elicit SIE against a cointroduced TCV replicon, and that it likely accomplished this by sequestering freshly translated p28 molecules into self-perpetuating p28 polymers. These earlier observations raised at least three questions. (i) Does the SIE activity of p28 require any specific domains or amino acid residues in p28? (ii) Can this replication-repressing SIE activity of p28 be decoupled from its essential role in replication? (iii) Can either of the p28 functions (replication and SIE) be decoupled from the RdRP activity of $\mathrm{p} 88$ ? This last question is of interest because p88 encompasses the entire p28 at its $\mathrm{N}$ terminal 2/5 (Fig. 1A). The current study addresses these questions by identifying a 12-amino-acid (aa) region in p28 that is required for efficient SIE. Moreover, two single-amino-acid changes within this region enabled not only the decoupling of SIE activity of p28 from its replication function but also the separation of the replication function of p28 from that of $\mathrm{p} 88$. Finally, both single-amino-acid mutations lowered the cellular concentration of p28, and diminished the number of p28 inclusion bodies. Our findings unveil novel mechanistic details of SIE elicitation by TCV p28, and provide powerful new tools for further elucidation of TCV SIE.

\section{RESULTS}

Deletion mutagenesis of p28-GFP fusion protein identifies a small region needed for robust SIE elicitation.

We recently reported that a C-terminal GFP fusion bolsters the ability of p28 to elicit SIE to TCV (Zhang et al. 2017). Specifically, expression of the p28-GFP fusion protein in $\mathrm{Ni}$ cotiana benthamiana cells abolished the replication of TCV_sg2R, an mCherry-expressing TCV replicon, in the same cells, erasing the replication-dependent mCherry fluorescence (Zhang et al. 2017). Additionally, the strong replicational repression by p28-GFP correlated with the occurrence of large, intense green fluorescent foci in these cells (Zhang et al. 2017). These easy-to-monitor fluorescent phenotypes prompted us to use p28-GFP as the launch pad to map domains in p28 essential for SIE elicitation. To systematically screen for these domains, we generated a series of in-frame deletions within the p28 portion of p28-GFP (Fig. 2A). The effect of these deletions on the SIE-eliciting ability of p28 was evaluated by expressing the mutant proteins in $N$. benthamiana cells and assessing the replication of the cointroduced TCV_sg2R replicon with Northern blot hybridizations. In addition, we also monitored the replication-dependent diffuse mCherry fluorescence, as well as the GFP-fluorescent mutant fusion proteins, using confocal microscopy.

Deletion of the N-terminal half of the 250-aa p28 (mutant $\Delta 2-125$ ) caused only minimal loss in the SIE elicitation by $\mathrm{p} 28$ -
GFP (Fig. 2B), leading to a very modest recovery of TCV_sg2R replication (Fig. $2 \mathrm{~A}$ and $\mathrm{B}$, lanes 7 and 8 ). A specific $\mathrm{N}$ terminal domain could not be delineated because none of the smaller deletions within this region, including $\Delta 2-36, \Delta 37-50$, $\Delta 37-100, \Delta 51-100$, and $\Delta 76-125$, led to detectable replication of TCV_sg2R (Fig. 2B, lanes 11 to 18, and C, lanes 11 and 12). These results suggest that, in the presence of the C-terminal GFP tag, the N-terminal half of p28 plays a modest role in SIE elicitation. Consistent with this conclusion, the $\Delta 2-100$ deletion had an even smaller effect than $\Delta 2-125$ on SIE, leading to only occasional detection of TCV_sg2R replication (Fig. 2B, lanes 9 and 10 , and $\mathrm{D}$, lanes 7 and 8 ).

Interestingly, the $\Delta 2-155$ mutant, which extends the $\Delta 2-125$ deletion for just 30 aa, permitted a substantially higher level of TCV_sg2R replication (Fig. 2C, compare lanes 7 and 8 with 9 and 10). However, a separate deletion of the 30 aa alone

A TCV:

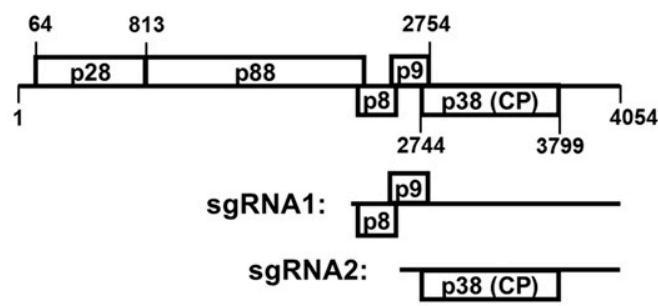

B
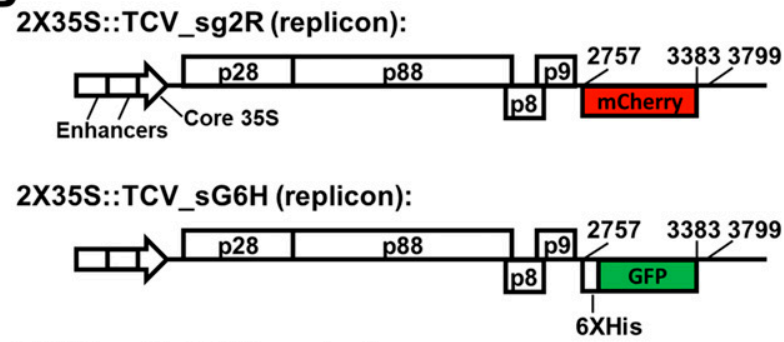

2X35S::p28-GFP (transient):
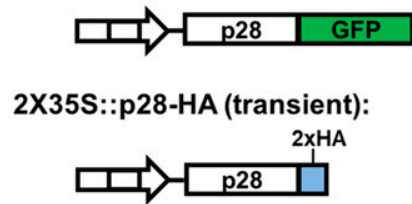

2X35S::p28 (tag-free, transient):

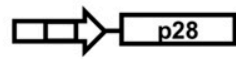

Core35S::p88 (tag-free, transient):

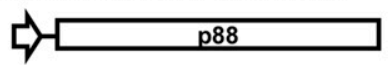

Fig. 1. Turnip crinkle virus (TCV) genome organization, and the configurations of the constructs used in this study. A, Organization of the 4,054nucleotide single-stranded, positive sense (+) TCV genome. Various boxes with labels within (e.g., p28, p88, p8, p9, and p38) denote the proteins encoded by the TCV genome, with the boundaries of some open reading frames shown. The three $3^{\prime}$ proximal proteins (p8, p9, and $\mathrm{p} 38$ ) are translated from two subgenomic RNAs (sgRNA1 and 2). B, Representative constructs used in this study. Most constructs were equipped with the strong $35 \mathrm{~S}$ promoter with the enhancer duplicated $(2 \mathrm{X} 35 \mathrm{~S})$ in order to ensure efficient transcription. Only the last, p88-expressing construct was driven by the relatively weak core $35 \mathrm{~S}$ promoter because a high level of p88 translation was shown to repress TCV replication (Zhang et al. 2019). The topmost construct is a replicon shown previously to launch TCV replication in plant cells, leading to mCherry expression from the sgRNA2 (Cao et al. 2010; Zhang et al. 2017). All other constructs direct transient expression of the respective proteins in the absence of TCV replication. GFP $=$ green fluorescent protein 
$(\Delta 126-155)$ had a much weaker though consistently detected release of TCV_sg2R replication (Fig. 2C, compare lanes 13 and 14 with lanes 7 and 8). This suggested that loss of SIE activity by the mutant $\Delta 2-155$ could be attributed to the additive or synergistic effect of two regions: amino acids 2 to 125 and 126 to 155 . Nevertheless, specific SIE-eliciting sequence motifs or amino-acid residues within the first 155 -aa region could not be reliably identified with the GFP-tagged constructs.
A

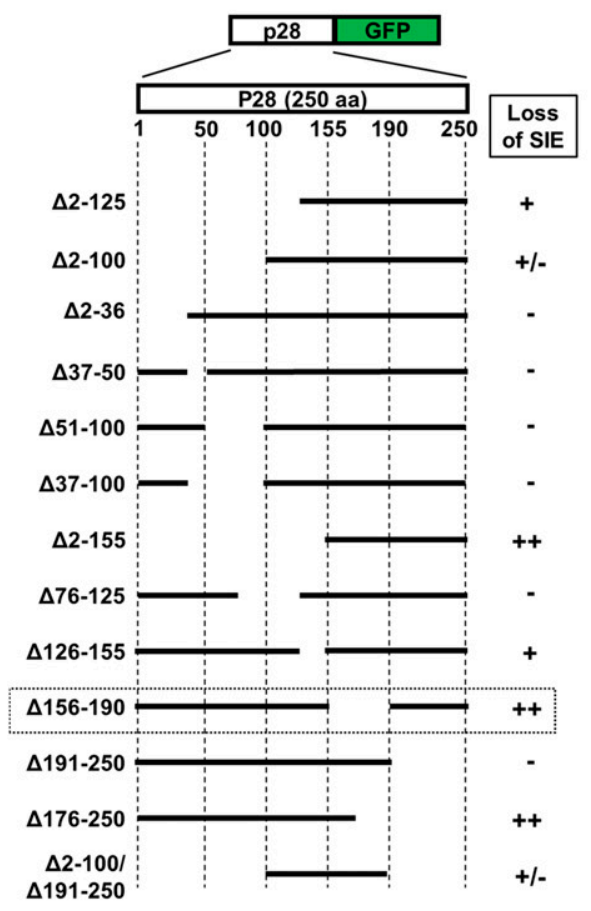

B

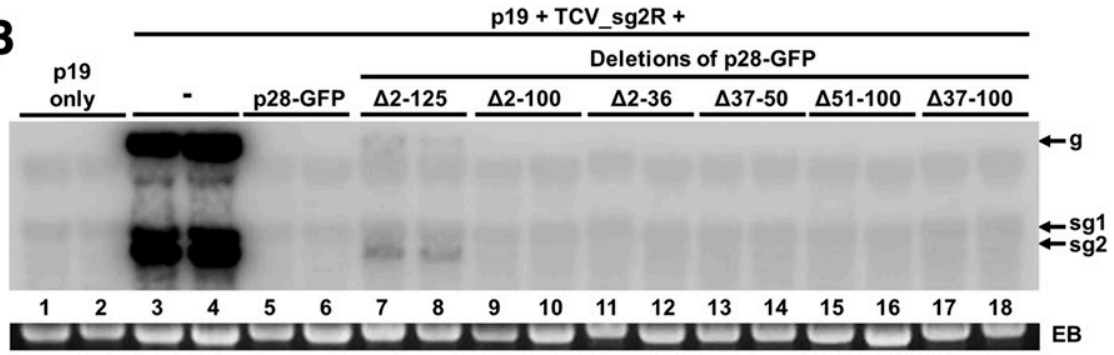

C

p19 + TCV_sg2R +

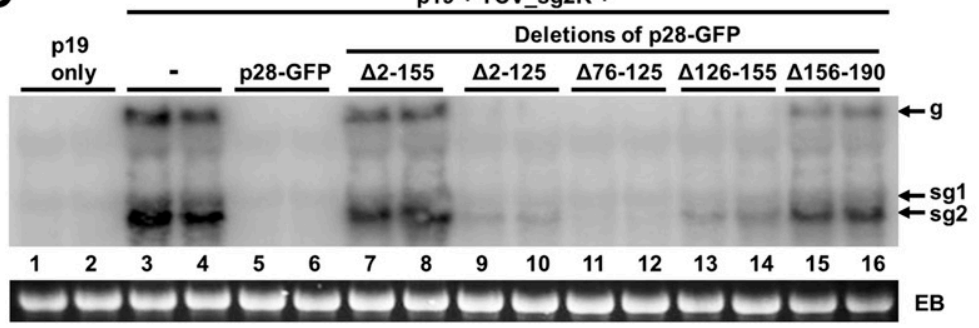

D

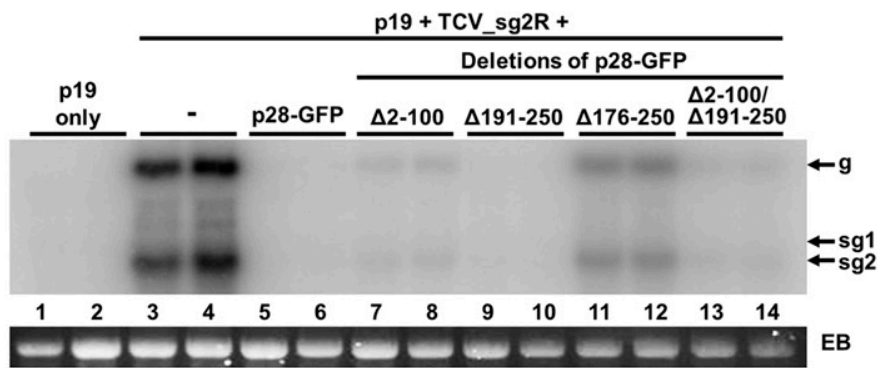

$\mathbf{E}$
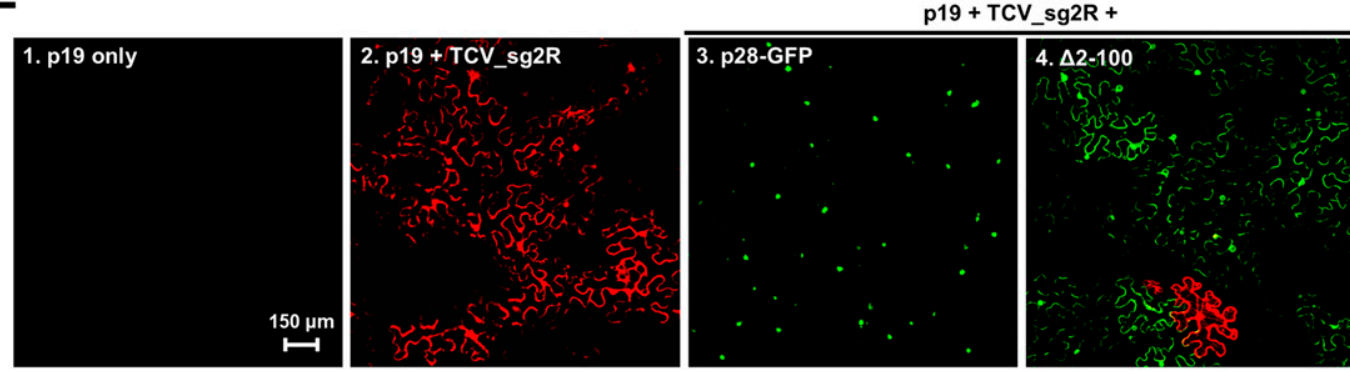

p19 + TCV_sg2R +
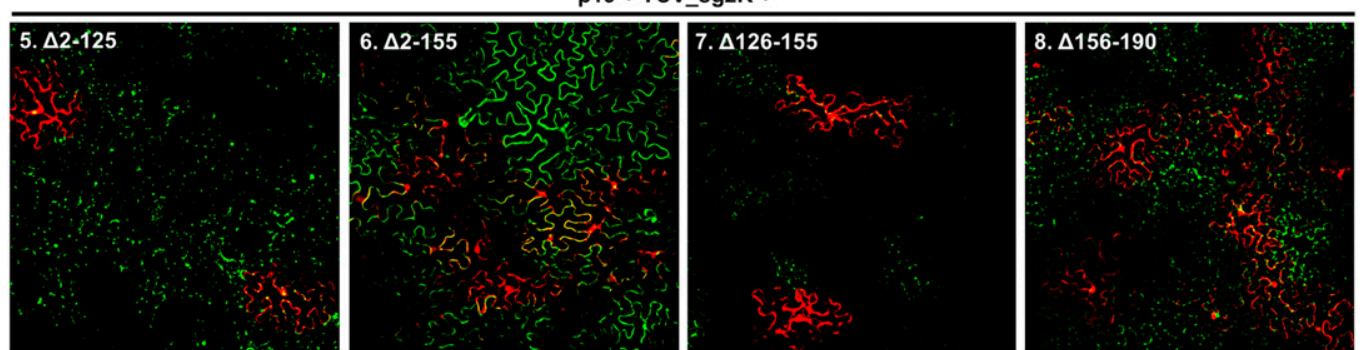

Fig. 2. Deletion mutagenesis on the p28-green fluorescent protein (GFP) backbone identifies the 156- to-190-amino-acid region as needed for robust superinfection exclusion (SIE). A, Diagrams of deletion mutants of p28-GFP showing their respective deleted regions. B, C, and D, Northern blotting results showing the levels of replicational repression imposed on the TCV_sg2R replicon by p28-GFP and its deletion mutants. The p19 silencing suppressor was included to mitigate the impact of RNA silencing. The double loadings represented two independent samples extracted from different leaves. The positions of genomic RNA and subgenomic RNA1 and 2 were highlighted with arrows (g, sg1, and sg2, respectively). EB = ethidium bromide-stained gel. E, Confocal images of Nicotiana benthamiana leaf cells treated with select p28-GFP deletion mutants along with the TCV_sg2R replicon. 
Surprisingly, another relatively small deletion $(\Delta 156-190,35$ aa in total) caused a loss of SIE activity that rivaled the large $\Delta 2-155$ deletion (Fig. 2C, compare lanes 15 and 16 with 7 and 8), thus identifying a region with a relatively strong effect. Beyond amino acid 190, the entire C-terminal 60-aa region could be removed without detectably compromising the SIEeliciting activity of p28-GFP (Fig. 2D, $\Delta 191-250$, lanes 9 and 10). Indeed, a double deletion mutant combining the $\Delta 2-100$ and $\Delta 191-250$ deletions was no worse than $\Delta 2-100$ alone at repressing TCV_sg2R replication (Fig. 2D, compare lanes 13 and 14 with lanes 7 and 8). This further confirmed that the last 60-aa region was dispensable for SIE elicitation by p28-GFP.

Notably, the loss of SIE activity by many of the deletion mutants was accompanied by dramatic changes in the intracellular behavior of mutant proteins. For example, although the SIE loss caused by the $\Delta 2-100$ deletion was minimal, the $\Delta 2-100-G F P$ formed large foci in substantially fewer cells than the wild-type p28-GFP (Fig. 2E, images 3 and 4) and, instead, exhibited a distribution resembling soluble GFP in many cells (Fig. 2E, image 4). Nevertheless, the smaller, more numerous foci formed by the GFP-tagged $\Delta 2-125, \Delta 126-155$, and $\Delta 156$ 190 proteins appear to be consistent with a requirement for larger aggregates in SIE elicitation (Fig. 2E, images 5, 7, and $8)$. Further supporting this idea is the observation that the $\Delta 2$ 155-GFP mutant protein was predominantly diffusely distributed, suggestive of a soluble protein (Fig. 2E, image 6).

\section{Additional deletions within the 156- to-190-aa region identify a 12-aa stretch with a significant role in eliciting SIE.}

That a substantial loss of SIE-eliciting activity could be inflicted by a mere 35 -aa deletion $(\Delta 156-190)$ prompted us to further interrogate this small region using the p28-GFP fusion construct. To this end, three smaller deletions $(\Delta 156-170, \Delta 171-$ 182 , and $\Delta 183-190$, deleting 15,12 , and 8 aa, respectively) were generated and the resulting deletion mutants were analyzed for their ability to elicit SIE against TCV_sg2R. Neither $\Delta 156-170$ (Fig. 3A, lanes 9 and 10) nor $\Delta 183-190$ (Fig. 3A, lanes 13 and 14) caused detectable loss of the SIE-eliciting activities. By contrast, deletion of amino acids 171 to 182 appeared to account for most of the SIE loss observed with the $\Delta 156-190$ mutant.

We next investigated whether this 12 -aa region contained any specific amino-acid residue(s) critical for SIE elicitation by generating three consecutive 4-aa deletions. All three deletion mutants (Fig. 3B, lanes 9 to 14, ) compromised the SIE-eliciting activity of p28-GFP to extents similar to the 12-aa deletion mutant $\Delta 171-182$ (Fig. 3B, lanes 7 and 8). Therefore, it appears that the entire 12-aa region is needed for eliciting a robust SIE. We then chose the last four amino-acid residues of this region for single-amino-acid mutagenesis and changed each of them to alanine (Fig. 3B, lanes 15 to 22). When these single-amino-acid mutants were introduced into plant cells along with the TCV_sg2R replicon, only the F182A mutant caused a barely detectable accumulation of TCV_sg2R RNAs (Fig. 3B, lanes 21 and 22), suggesting that the phenylalanine to alanine change weakly compromised the SIE-eliciting ability of p28-GFP.

Confocal microscopy showed that $\Delta 156-170, \Delta 171-182$, and $\Delta 183-190$ all abolished the ability to form large aggregates (Fig. 3C, images 3 and 4; Fig. 2E) and, instead, formed smaller but more numerous foci that sometimes arranged along cell boundaries. The $\Delta 156-170$ mutant was noteworthy because it did allow occasional escape of red fluorescent cells and, thus, TCV_sg2R replication, even though such low-level replication was not detected with Northern blotting (discussed above). This suggested that it was important to use both Northern blotting and confocal microscopy to capture the full spectrum of SIEeliciting activities by these mutants.
Interestingly, the smaller 4-aa deletion mutant $\Delta 179-182$ and the larger 12-aa deletion mutant $\Delta 171-182$ behaved highly similarly when inspected with confocal microscopy. Both permitted TCV_sg2R replication in easily detectable numbers of cells, even though their replication levels as measured with Northern blotting were much lower than controls without any p28-GFP derivatives (Fig. 2B, lanes 7 to 14). Finally, three of the four single-amino-acid mutants (P179A, R180A, and V181A) formed large aggregates very similar to those of wildtype p28-GFP, though smaller foci were also seen (Fig. 3C, images 7 to 9). By contrast, in cells treated with the F182A mutant, large aggregates were absent (Fig. 3C, image 10). Among these cells, replication-dependent red fluorescence was occasionally observed, thus confirming the weak TCV_sg2R replication detected with Northern blotting (Fig. 2B, lanes 21 and 22).

\section{Replacing the C-terminal GFP with a double hemagglutinin tag unveils additional regions important for SIE-elicitation by $\mathbf{p 2 8}$.}

Experiments using the p28-GFP fusion protein mutants identified the 12-aa region spanning amino acids 171 to 182 as critically important for p28 to elicit SIE. However, they failed to identify any other p28 regions that were specifically needed for SIE elicitation. These results contrasted with our previous observations implicating the $\mathrm{N}$ terminus of p28 in SIE elicitation. We showed earlier that fusing a short G11 tag (25 aa) at the $\mathrm{N}$ terminus rendered p28 incapable of eliciting SIE (Zhang et al. 2017). We also found that a truncated form of p28 missing the first 36 aa was no longer able to elicit SIE (Zhang et al. 2017; Zhang et al. 2019). Did the C-terminal GFP fusion in p28-GFP allow p28 to circumvent certain other critical regions, possibly through the tendency of GFP to self-dimerize (Shaner et al. 2005)? To address this question, we decided to adopt $\mathrm{p} 28$ hemagglutinin (HA), another strong SIE-eliciting p28 variant with a C-terminal, duplicated HA tag (Zhang et al. 2017; Zhang et al. 2019). To this end, the GFP in all deletion mutants described earlier was replaced with the HA tag and the resulting HA-tagged mutants were retested for SIE induction.

The HA substitution revealed a role for the N-terminal region in SIE elicitation (Fig. 4A). The SIE-eliciting activity of p28HA was not detectably weakened by deleting amino acids 51 to 100 or 191 to 250 , suggesting that up to 110 aa residues of p28 could be removed without detectably weakening SIE elicitation. However, this activity is clearly compromised by deletions of amino acids 2 to 36,126 to 155 , and 156 to 190 and, to lesser extents, amino acids 37 to 100,76 to 125 , and 183 to 190 . Therefore, use of p28-HA as the backbone permitted the identification of two additional p28 regions with potentially specific roles in SIE elicitation; namely, amino acids 2 to 50 and 126 to 155 .

\section{Use of p28-HA reveals key amino acid residues within the 171- to-182-aa region critical for SIE elicitation.}

We next attempted to fine map the 171- to-182 region, whose deletion compromised SIE in the GFP-tagged p28 variant, using the HA-tagged p28. Use of the p28-HA backbone revealed partial SIE losses caused by the $\Delta 156-170$ and $\Delta 183-190$ deletions (Fig. 4B, lanes 7, 8, 11, and 12) that were undetectable with the p28-GFP backbone (Fig. 3A, lanes 9, 10, 13, and 14). However, it is important to note that only the deletion of the 171- to-182-aa core sequence fully recapitulated the phenotype of the larger 156- to-190 deletion (Fig. 4B, compare lanes 5 and 6 with 9 and 10). Furthermore, each of the three smaller, 4-aa deletions $(\Delta 171-174, \Delta 175-178$, and $\Delta 179-182)$ within the 171 to-182-aa region inflicted an SIE loss that is nearly identical to the 12-aa $\Delta 171-182$ (Fig. $4 \mathrm{~B}$, lanes 13 to 18 ). 
We next reexamined the effect of four single-amino-acid substitutions using the p28-HA backbone. The very weak SIE loss caused by the F182A mutation in p28-GFP background now became easily detectable (Fig. 4C, lanes 15 and 16). Indeed, only the R180A mutation failed to inflict a meaningful loss in the SIE-eliciting activity of p28-HA (Fig. 4C, lanes 11 and 12). By contrast, both P179A and V181A mutations correlated with weak losses in SIE elicitation, with P179A being slightly less disruptive than V181A (Fig. 4C, lanes 9, 10, 13, and 14).

\section{TCV replicons containing the V181A mutation} overcome SIE in a substantial fraction of cells.

We next wondered whether the single-amino-acid mutations that compromised SIE in transiently expressed p28-GFP and
p28-HA had similar effects in TCV replicons. To resolve this question, we introduced the V181A and F182A mutations into TCV_sG6H and TCV_sg2R, two TCV replicons that encode GFP (sG6H denotes GFP with an N-terminal 6XHis tag) (Powers et al. 2008) and mCherry, respectively, in place of the coat protein (Fig. 1). These replicons were then tested in coinfected cells to determine whether the mutations compromised SIE of TCV.

Although the GFP-encoding replicon (TCV_sG6H) appeared to replicate less robustly and produced much less sgRNA (Fig. $5 \mathrm{~A}$, lane 2), it produced easily detectable green fluorescence (Fig. 5B, top left), probably due to translational initiation from an internal ribosomal entry site identified recently (May et al. 2017). Replicons with the V181A mutation (V181A_sG6H and V181A_sg2R) replicated to levels comparable with wild-type

A

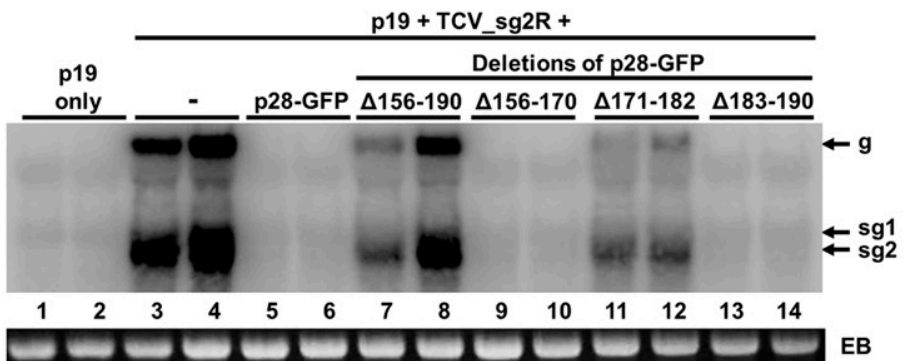

B

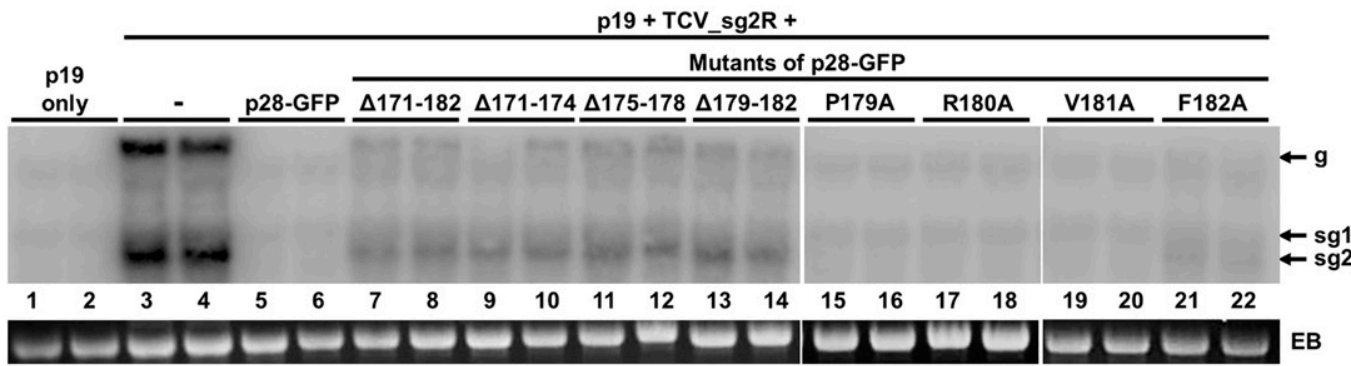

C
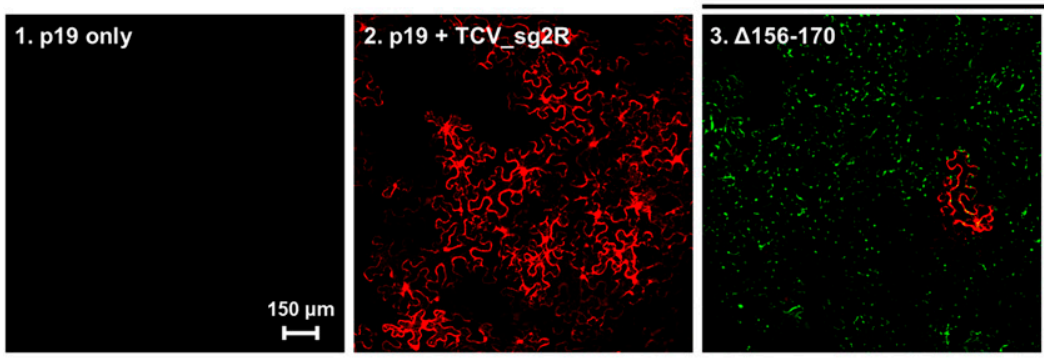

p19 + TCV_sg2R +
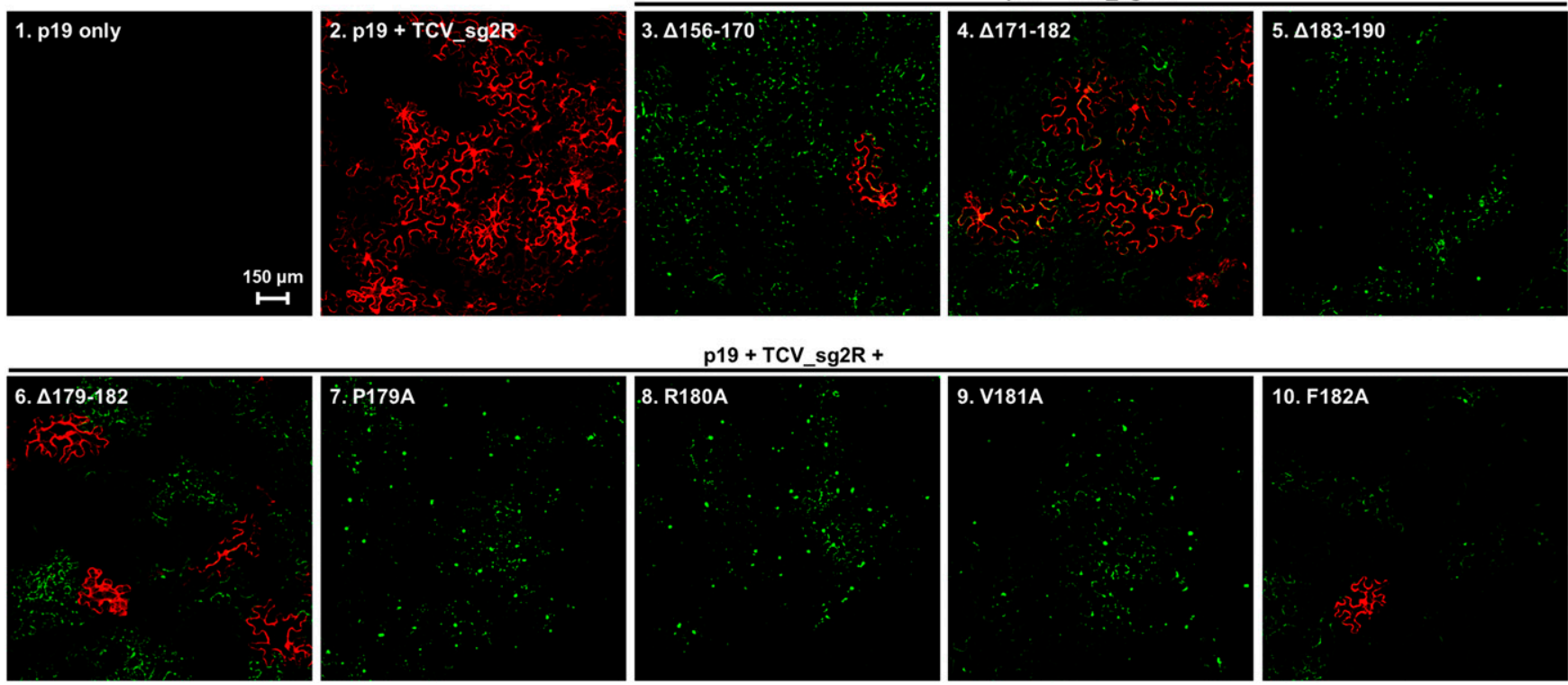

p19 + TCV_sg2R +

Fig. 3. Mapping the 156- to-190-amino-acid region of p28-green fluorescent protein (GFP) with smaller deletions and single-amino-acid exchanges identifies amino acids 171 to 182 as the key determinant of superinfection exclusion. A and B, Northern blots showing the levels of replicational repression imposed on the TCV_sg2R replicon by various smaller deletion and single-amino-acid mutants of p28-GFP. Rows g, sg1, g2, and EB = genomic RNA, subgenomic RNA1 and 2, and ethidium bromide-stained gel, respectively. C, Confocal images of Nicotiana benthamiana leaf cells treated with select p28-GFP mutants along with the TCV_sg2R replicon. 
replicons encoding wild-type p28 (and p88) (Fig. 5A, compare lanes 4 and 5 with 2 and 3, respectively). By contrast, the F182A mutation abrogated TCV replication to levels below the detection limit of Northern blotting (Fig. 5A, lanes 6 and 7). Next, the V181A replicons were subjected to coinfection experiments to assess the levels of SIE. Note that coinfections of replicons encoding the same p28 variant but different fluorescent proteins did not affect their replications (Fig. 5A, lanes 8 to 13). Although wild-type replicons exhibited strong SIE that precluded the coexpression of GFP and mCherry in the same cells (Fig. 5B, top row), the V181A replicons permitted such coexpression in approximately $19 \%$ of green- or red-fluorescent cells. These results demonstrated that the same V181A mutation that compromised the ability of p28-HA to repress TCV replication also compromised the ability of p28 to elicit SIE in TCV replicons.
The F182A mutation disrupts

the replication function of $\mathbf{p} 28$ but not $\mathrm{p88}$.

The impact of the F182A mutation on replicon SIE could not be readily examined because it abolished TCV replication when incorporated in replicons (Fig. 5). Because the p28 coding sequence is shared by p88 in the replicon context, the F182A change could have disrupted the replication function of $\mathrm{p} 28$, or $\mathrm{p} 88$, or both. To resolve this issue, we attempted to complement the replication of the F182A_sg2R replicon using transiently expressed, tag-free p28 and p88 (Fig. 1B). F182A $\mathrm{sg} 2 \mathrm{R}$ replication was restored to easily detectable levels by transiently expressed p28 (Fig. 6A, compare lanes 9 and 10 to 5 and 6) but not p88 (Fig. 6A, lanes 13 and 14). Therefore, the F182A mutation primarily disrupted the auxiliary replication function of $\mathrm{p} 28$. Although minor perturbation of $\mathrm{p} 88$ function by F182A could not be ruled out, such perturbation did
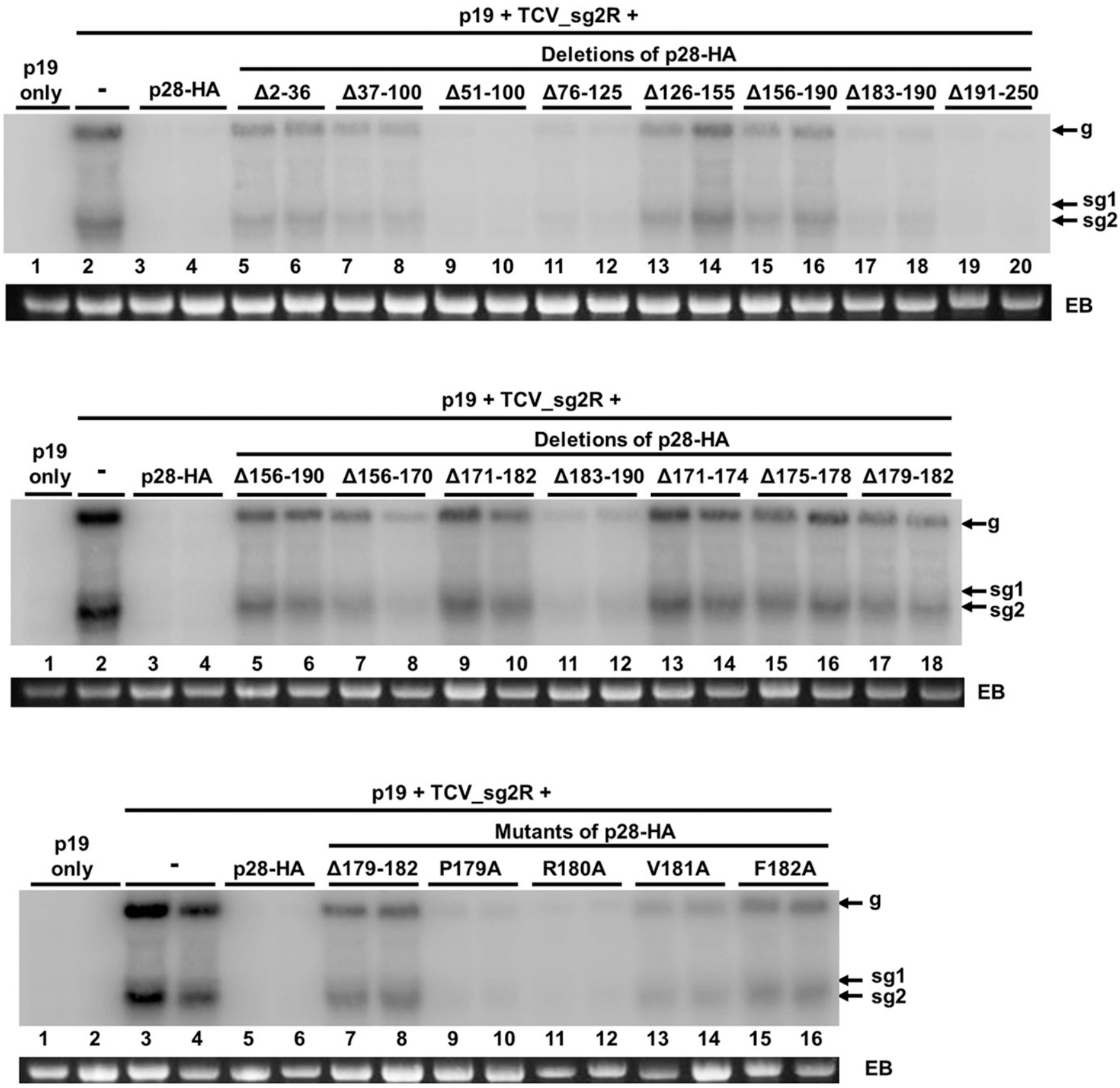

Fig. 4. Use of the p28-hemagglutinin (HA) backbone reveals additional regions contributing to superinfection exclusion elicitation by p28. All panels are Northern blots showing the levels of replicational repression imposed on the TCV_sg2R replicon by various mutants of p28-HA. Rows g, sg1, g2, and EB = genomic RNA, subgenomic RNA1 and 2, and ethidium bromide-stained gel, respectively. 
not abolish TCV replication. Taken together, the F182A mutation severely debilitated the replication function of p28 but not $\mathrm{p} 88$. In conclusion, this mutation decouples the replication functions of p28 and p88.

An F182A-containing replicon, upon replicational complementation, exerts robust SIE against a wild-type replicon.

We next examined whether the replicational defect of the F182A-containing replicons could be complemented by another replicon encoding a replication-competent $\mathrm{p} 28$, and whether the p28(F182A) protein produced by the complemented replicon might exert SIE against the replicon that provided the complementing p28. To answer this question, we paired the F182A_sg2R replicon with TCV_sG6H or V181A_sG6H, in coinfections of $N$. benthamiana cells. Both TCV_sG6H and V181A_sG6H replicons complemented the F182A defect (Fig. $6 \mathrm{~B}$, images 4 and 6), enabling an approximately 10-fold increase in the number of cells replicating F182A_sg2R (Fig. 6B, images 3,4 , and 6 , and $\mathrm{C}$, red bars in groups 3,4 , and 6 ).

However, complementation by the two GFP-encoding replicons led to starkly different SIE phenotypes. The complementation by TCV_sG6H, which encodes a wild-type p28, gave rise to mCherry-expressing cells that never contained GFP fluorescence (Fig. 6B, image 4, and C, group 4). Conversely, the GFP-expressing cells also never contained mCherry. Because the TCV_sG6H construct must reside in the same cells as the F182A_sg2R construct in order to supply the replicationcomplementing wild-type p28, these observations strongly suggested that the $\mathrm{p} 28(\mathrm{~F} 182 \mathrm{~A})$ protein, now produced by the replicating F182A_sg2R replicon, was able to exert a powerful SIE to block TCV_sG6H replication. Two converging factors could explain this outcome: (i) the wild-type p28 produced by the nonreplicating TCV_sG6H transcript could form small aggregation cores in certain parts of the cell or (ii) replicationdependent production could cause p28(F182A) mutant protein

A
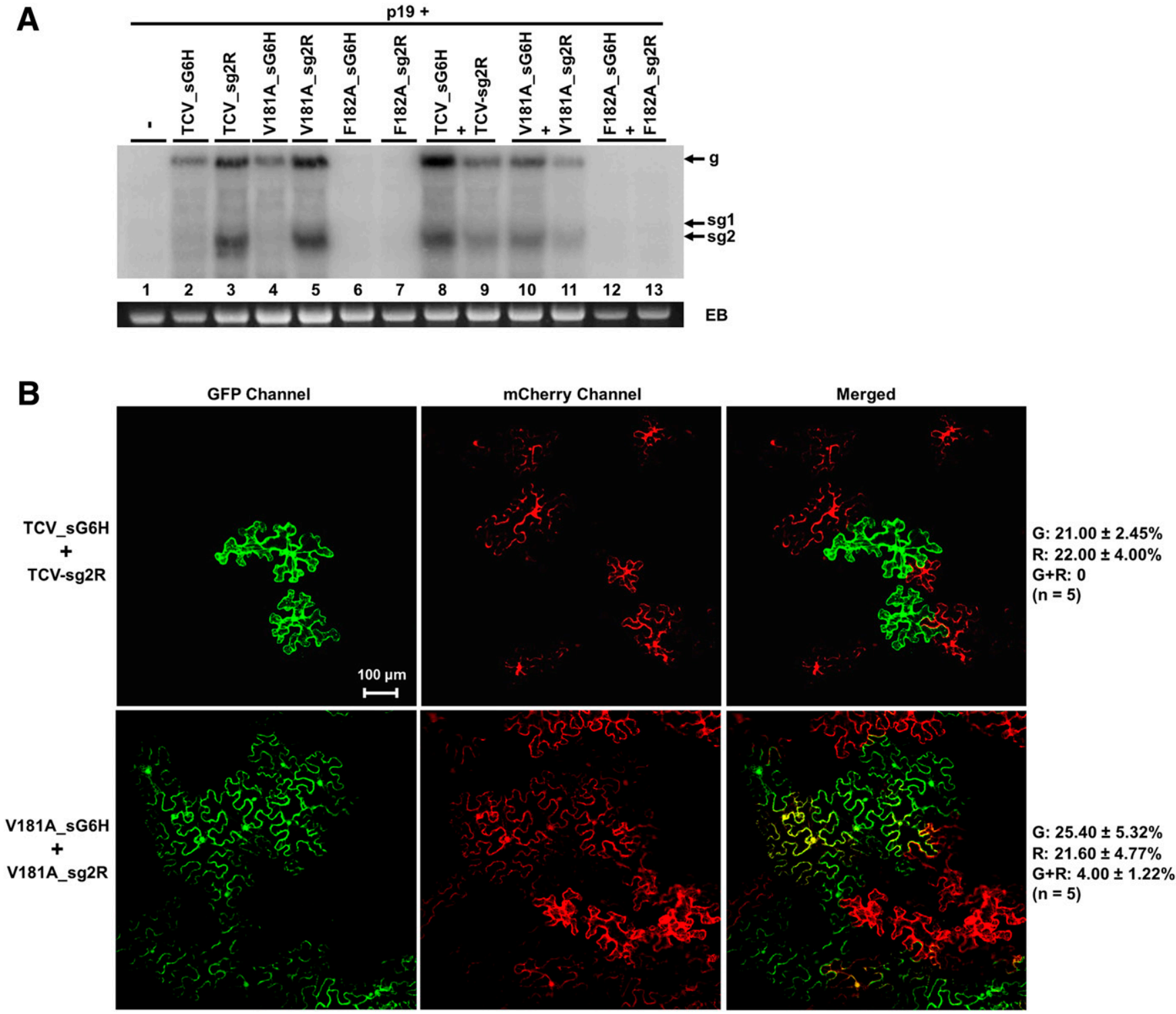

R: $22.00 \pm 4.00 \%$

G+R: 0

$(n=5)$
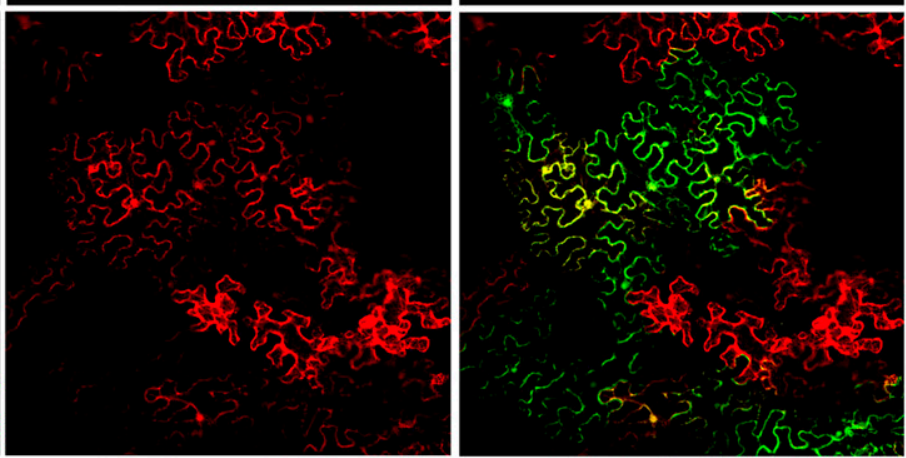

G: $25.40 \pm 5.32 \%$

R: $21.60 \pm 4.77 \%$

G+R: $4.00 \pm 1.22 \%$

$(n=5)$

Fig. 5. Replication and superinfection exclusion (SIE) of mutant replicons with the V181A and F182A mutations. A, Northern blot showing the replication (lanes 4 to 7) and SIE (lanes 10 to 13) of the mutant replicons encoding different fluorescent proteins (green fluorescent protein [GFP] or mCherry). Rows g, $\mathrm{sg} 1, \mathrm{~g} 2$, and EB = genomic RNA, subgenomic RNA1 and 2, and ethidium bromide-stained gel, respectively. B, Confocal images showing robust SIE between two wild-type replicons (top images) and the partially compromised SIE between the two V181A mutant replicons. The quantification was based on five independent leaf areas, each containing 40 to 50 cells. $\mathrm{G}=\mathrm{GFP}$-fluorescent cells, $\mathrm{R}=$ mCherry-fluorescent cells, and $\mathrm{G}+\mathrm{R}=$ cells containing both GFP and mCherry. 
to accumulate to very high levels, effectively overcoming the poor stability caused by the mutation (discussed below).

The assertion that both F182A_sg2R and TCV_sG6H constructs must have been present in those red fluorescent cells is further bolstered by the contrasting SIE phenotype observed from the complementation mediated by V181A_sG6H (Fig. $6 \mathrm{~B}$, image 6 , and $\mathrm{C}$ ), because these experiments were carried out in parallel. Specifically, when the replication of F182A_sg2R

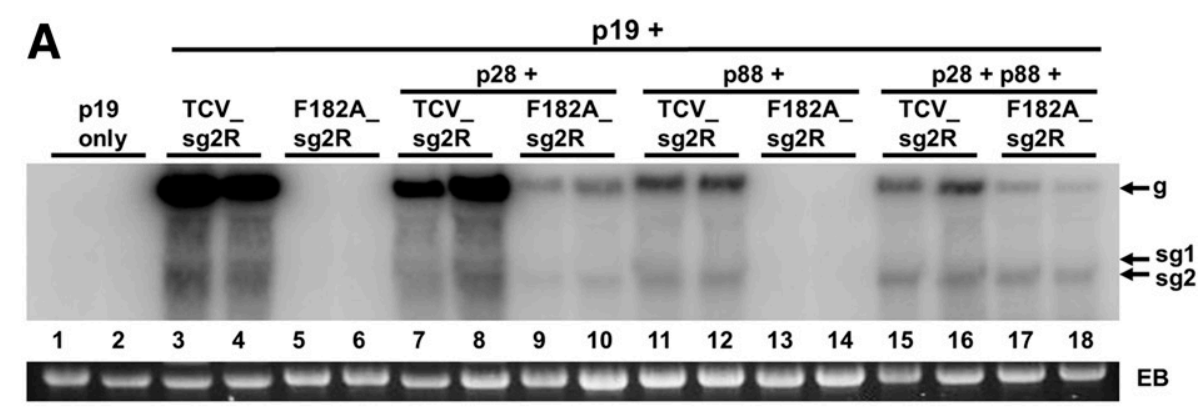

B
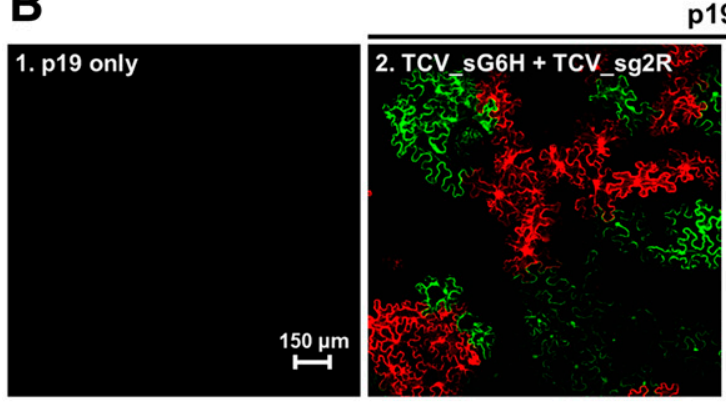

p19+

p19+
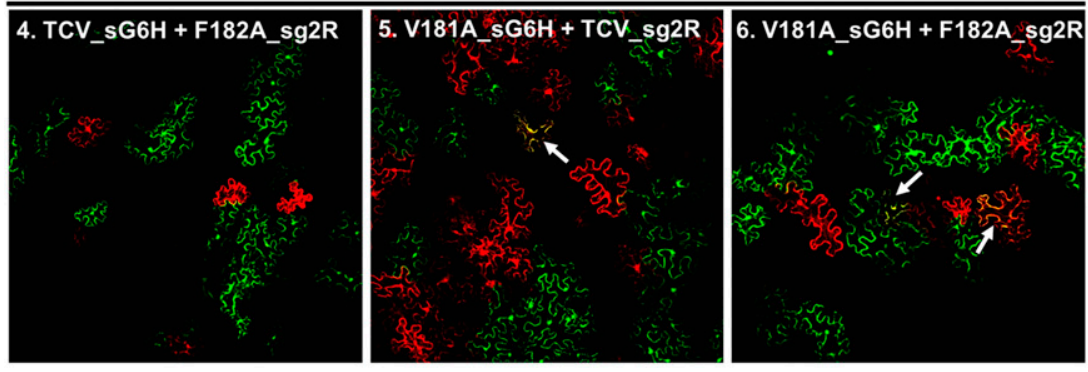

C

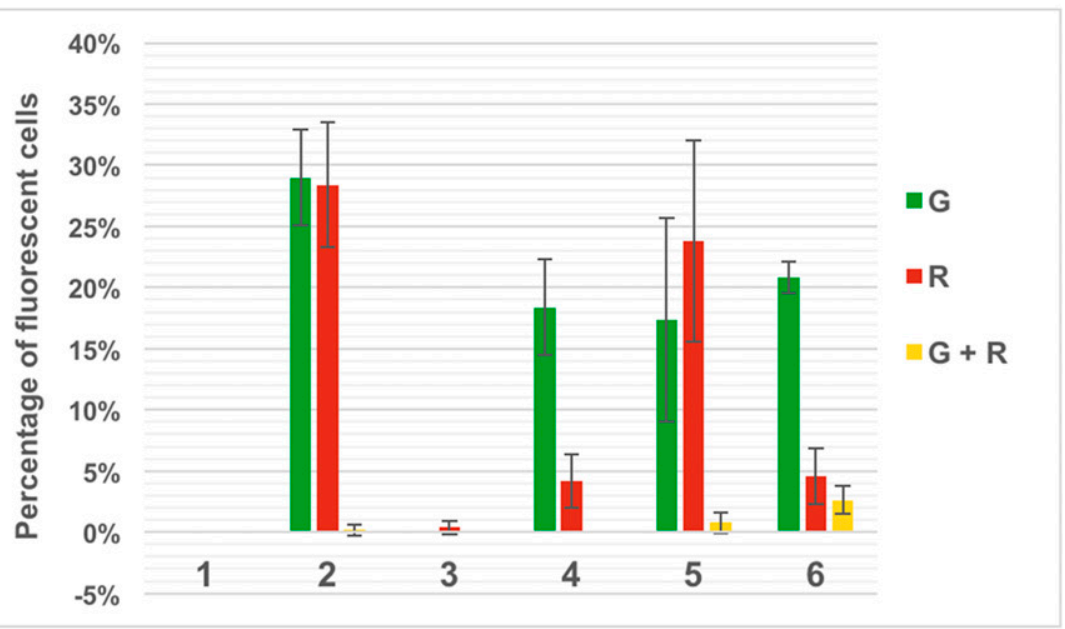

Fig. 6. In replicons, the F182A mutation selectively abrogates the replication function of p28 but not its ability to elicit superinfection exclusion (SIE). A, Northern blot showing that the replicational defect of the F182A replicon was complemented by transiently expressed p28 but not p88. Rows g, sg1, g2, and EB = genomic RNA, subgenomic RNA1 and 2, and ethidium bromide-stained gel, respectively. B, Confocal images showing that the replicational defect of the F182A replicon was also complemented by cointroduced wild-type and V181A replicons, and the successfully complemented F182A replicon exerted robust SIE against the wildtype replicon. C, Quantification of replicational complementation and SIE of the F182A replicon. The numbering of the bar groups corresponds to that of images in B. $\mathrm{G}=$ green fluorescent protein (GFP)-fluorescent cells, $\mathrm{R}=$ mCherry-fluorescent cells, and $\mathrm{G}+\mathrm{R}=$ cells containing both $\mathrm{GFP}$ and $\mathrm{mCherry}$. In all sets, leaf areas of the same size, containing 40 to 50 cells each, were chosen from five different leaves and counted for cells with different fluorescence (GFP, mCherry, or both). 
was complemented with the V181A_sG6H replicon, approximately one-half of the red fluorescent cells also contained GFP fluorescence. This probably reflected the partial loss of SIEeliciting capacity by the $\mathrm{p} 28(\mathrm{~V} 181 \mathrm{~A})$ protein (Figs. 5B and 6B, image 5). However, this result also demonstrated that two different replicon constructs readily entered the same agroinfiltrated cells. Together, these results indicated that, when expressed from a replicating replicon, the $\mathrm{p} 28(\mathrm{~F} 182 \mathrm{~A})$ protein is fully capable of exerting a strong SIE against another replicon in the same cells, despite its inability to facilitate replication on its own. Therefore, in addition to decoupling the replication function of $\mathrm{p} 28$ and $\mathrm{p} 88$, the F182A mutation also decoupled the SIE function and replication function of p28-but in a manner diametrical to the V181A mutation.

\section{The p28(V181A) and p28(F182A) mutant proteins accumulated to substantially lower levels in cells and formed fewer $\mathbf{p} 28$ foci.}

Why did the V181A mutation weaken SIE in both transiently expressed p28 proteins and TCV replicons, whereas F182A did so only in transiently expressed p28? To address this question, we assessed the accumulation levels of the mutant $\mathrm{p} 28$ proteins using Western blotting. We chose to assay the GFP and HAtagged forms of p28 because a p28-specific antibody is not yet available, despite repeated attempts using multiple vendors. Both GFP and HA-tagged forms of V181A and F182A mutant p28 accumulated to levels substantially lower than their wildtype p28 counterparts (Fig. 7A and B). Importantly, although the HA-tagged forms of both mutant proteins diminished to levels below the detection limit (Fig. 7B), the GFP-tagged forms showed a slight difference between the two, with V181A but not F182A being marginally detectable. These results suggested that both mutations compromised p28 accumulation but the effect of F182A was more pronounced.

To further investigate how these two mutants handicapped SIE, we adopted a low-background bimolecular fluorescence complementation (BIFC) assay (Gookin and Assmann 2014; Kodama and $\mathrm{Hu} 2010$ ) to examine the intermolecular interactions among the mutant p28 proteins. This improved BIFC assay used a monomeric Venus (mVenus) protein that is split between the 10th and 11th (the last) $\beta$-strands to minimize selfassociation between the untagged fragments of this fluorescent protein, hence drastically reducing false positives. An additional advantage of this new system is that the large $\mathrm{N}$-terminal mVenus fragment (NVen, $10 \beta$-strands) could be readily detected using a GFP antibody. To prepare for the BIFC assay, the V181A and F182A mutants of p28, along with wild-type p28, were fused to the $\mathrm{N}$ termini of NVen and CVen (the 11th $\beta$-strand of mVenus). The resulting binary constructs were delivered into $N$. benthamiana cells to detect potential intermolecular interactions. As a negative BIFC control, we paired the NVen-tagged p28 with CVen-tagged p60, a deletion mutant of p88 with its N-terminal p28 portion removed. p28 and p60 did not interact with each other in preliminary BIFC assays (also discussed below).

To ensure that the constructs expressed the intended proteins, we first examined the accumulation levels of the NVen-tagged proteins (the CVen-tagged forms could not be detected due to the small size of the tag). The wild-type p28-NVen accumulated to very high levels in the presence of p28-CVen but to a much more modest level in the presence of p60-CVen (Fig. 7C, lanes 2 and 3 ), suggesting that it was stabilized by p28-p28 interactions (discussed below). Consistent with the results of Figure 7A and B, substantially reduced accumulation was observed with both V181A-NVen and F182A-NVen proteins, and the reduction was more dramatic for F182A-NVen (Fig. 7C, lanes 4 and 5). Compared with the p28-NVen/p60-CVen negative control (Fig.
7C, lane 3), the p28-NVen protein was slightly increased by V181A-CVen but modestly reduced by F182A-CVen (Fig. 7C, lanes 6 and 8). Notably, presence of p28-CVen did not enhance the accumulation of either V181A-NVen or F182A-NVen (Fig. 7C, lanes 7 and 9), suggesting that their levels were regulated independently of the presence of wild-type $\mathrm{p} 28$.

When viewed under a confocal microscope, the p28$\mathrm{NVen} / \mathrm{p} 28-\mathrm{CVen}$ pair gave rise to intensely bright yellow foci that resembled the p28-GFP foci (Fig. 7D, image 2). By contrast, the p28-NVen/p60-CVen pair was completely devoid of any yellow fluorescent signals, indicating an absence of p28-p60 interaction (Fig. 7D, image 3). Interestingly, V181ANVen and V181A-CVen still interacted to form foci in easily detectable but visibly fewer numbers (Fig. 7D, image 4). By contrast, the F182A-NVen/F182A-CVen codelivery resulted in substantially fewer foci that were mostly smaller and dimmer (Fig. 7D, image 5). However, pairing F182A-CVen with p28NVen, or vice versa, partially restored the formation of bright yellow foci (Fig. 7D, images 8 and 9). This experiment was repeated multiple times with similar results. The number of foci in each of the interactions was then quantified by counting the foci in multiple equal-sized leaf sections containing 40 to 50 cells (details below) and plotting the counts on box plots (Fig. $7 \mathrm{E})$. The quantification revealed that the V181A mutant proteins interacted with each other (group 4), or wild-type p28 (groups 6 and 7) to yield modestly fewer fluorescent foci than p28-p28 interactions (approximately 20\% reduction). By contrast, the F182A-F182A interaction led to a reduction of approximately $80 \%$ in focus numbers (group 5). Notably, this reduction was partially reversed by changing one of the interacting partners to a wild-type p28 derivative (groups 8 and 9). Thus, the F182A mutant proteins interacted with each other very inefficiently, probably due to low protein concentrations, but were able to coalesce with the nucleated core formed by wild-type p28 to produce the large foci detectable with confocal microscopy. Importantly, the lower protein levels of V181A and F182A mutant $\mathrm{p} 28$ proteins, coupled with reduced numbers of p28 foci, strongly correlated with the weakened SIE elicitation by these mutant proteins.

\section{DISCUSSION}

SIE protects host cells from being sequentially infected by the same virus. Although use of SIE in controlling plant virus diseases has been extensively documented, its underlying molecular mechanism and evolutionary rationale remain poorly understood (Zhang and Qu 2016). Using the model virus TCV, we have previously established that SIE occurred inside individual superinvaded cells, and acted to repress the replication of the superinfecting viral genomes (Zhang et al. 2015, 2017). We further discovered that SIE in TCV infections is mediated by a single TCV-encoded protein, the p28 auxiliary replication protein, likely through the formation of a self-perpetuating polymeric state that sequesters homologous p28 monomers. This model raised the possibility that SIE exists to ensure that progeny genomes of a virus are excluded from additional rounds of replication, thus minimizing error accumulation within single cells (Zhang et al. 2018). By extension, cells invaded by many copies of the same virus simultaneously, frequently in the form of collective infectious units (AndreuMoreno and Sanjuán 2018; Chen et al. 2015; Mirabelli and Wobus 2018; Miyashita et al. 2015), likely also experience similar molecular events that ensure that only a small number of the entered genomes are allowed to replicate and be subjected to cellular level selections. If this is true, then viral mutants that weaken SIE are expected to relax this restriction, allowing more viral genomes to replicate for multiple cycles in 
the same cells, causing fast error accumulation in viral populations and, probably, swift collapse of these populations.

The current study was designed to test the predictions of our working hypothesis by first identifying mutations within TCV p28 that compromise SIE. Our systematic deletion mutagenesis of p28, using two different C-terminally tagged forms of p28 as the backbone, allowed us to narrow down the SIE-eliciting activity of p28 to three regions: amino acids 2 to 50,126 to 155 ,
A

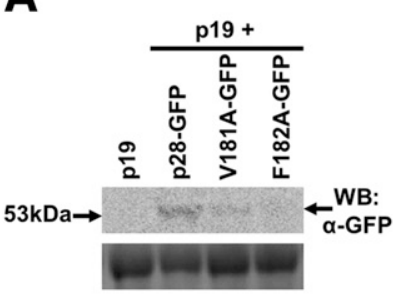

B

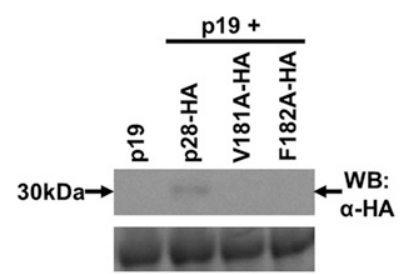

C

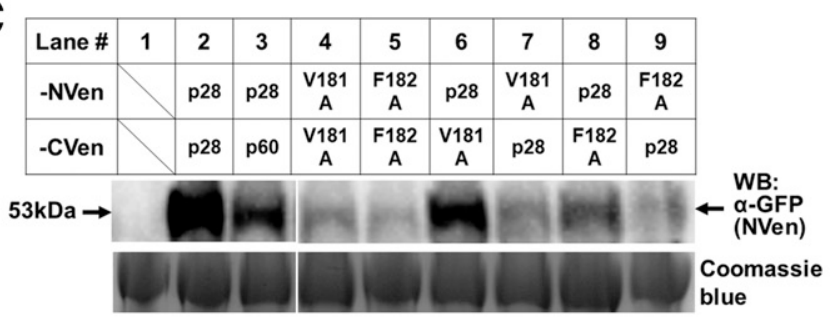

D
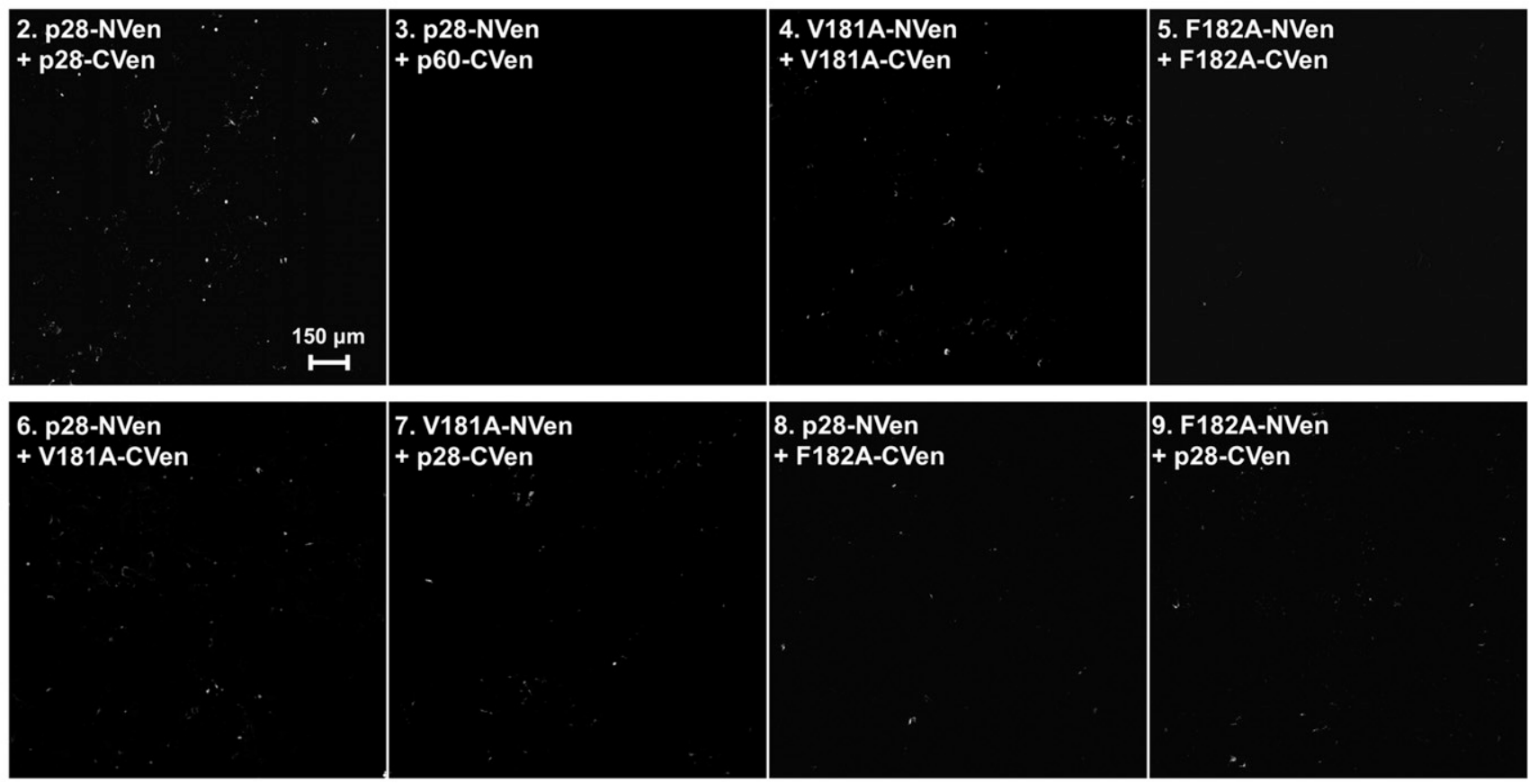

E

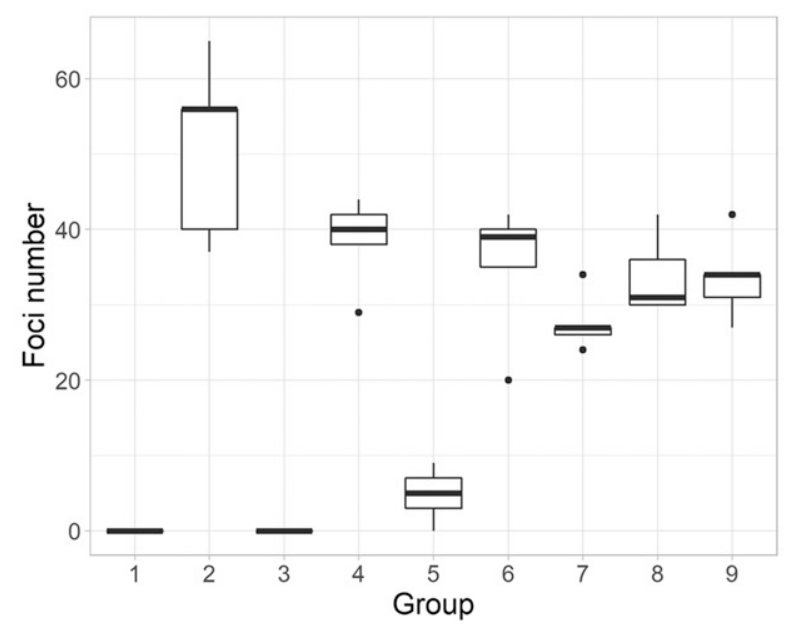

Fig. 7. V181A and F182A mutations reduced the level of transiently expressed p28 proteins and the number of polymeric foci formed by interacting p28 proteins. A, Western blot showing accumulation levels of green fluorescent protein (GFP)-tagged wild-type p28 protein and its V181A and F182A mutants. B, Western blot showing accumulation levels of hemagglutinin (HA)-tagged wild-type p28 protein and its V181A and F182A mutants. C, Western blot showing the accumulation levels of $\mathrm{N}$-terminal monomeric Venus fragment (NVen)-tagged p28, p28(V181A), and p28(F182A) in the presence of various 11th $\beta$-strand of monomeric Venus (CVen)-tagged interaction partners. D, Confocal images of multimeric p28 foci formed through p28-p28 interactions, visualized using monomeric Venus (mVenus)-based bimolecular fluorescence complementation (BIFC). The numbering of the images corresponds to that of lanes in C. E, Quantification of the number of $\mathrm{p} 28$ foci observed in various BIFC combinations. The numbering of the groups corresponds to that of lanes in C and images in D. 
and 156 to 190 . The 156 - to-190-aa region was then further interrogated because deletion of this region caused a consistent loss of SIE in both p28-GFP and p28-HA backgrounds. These efforts ultimately led to the identification of two amino acid residues-namely, valine $(\mathrm{V})$ and phenylalanine $(\mathrm{F})$ at positions 181 and 182, respectively-whose replacement by alanine (A) in replicons led to the decoupling of p28 replication function and its SIE elicitation function. It is interesting to note that these two residues appear to have different levels of conservation, with F182 but not V181 being highly conserved among the p28 analogs of multiple carmoviruses (not shown).

The lower protein levels of p28(V181A) correlated with weak SIE in both transient expressions and TCV replicons, suggesting that a protein concentration threshold had to be reached for $\mathrm{p} 28$ to elicit a robust SIE. This is consistent with the model proposed by us in earlier studies (Zhang et al. 2017, 2018). Why did the F182A mutant proteins, being even less abundant than V181A proteins, interfere with SIE elicitation only in the transiently expressed form but not in replicons? The answer might lie in the inability of the F182A-containing replicon to replicate by itself. Specifically, replication of the F182A mutant replicon must rely on wild-type (or V181A) p28 encoded by a different replicon (Fig. 6), and occurred in a small number of cells (approximately 5\%). In these cells, wild-type p28 protein was translated from the primary transcripts of the replicon construct but the replication of the wild-type replicon was delayed (Zhang et al. 2017). This delay created a precious window for the F182A mutant replicon to initiate replication with borrowed wild-type p28. Once it succeeded in replicating itself, the F182A mutant p28 would be produced in massive amounts, thus neutralizing the lower accumulation levels. More importantly, the presence of wild-type p 28 in these cells would facilitate the multimerization of p28 to form nucleation cores onto which the F182A p28 could coalesce, forming the large p28 foci that exert strong SIE. Indeed our BIFC experiments illustrated that the low self-interaction efficiency of the p28(F182A) protein was drastically improved when one of the interacting partners was changed to wild-type p28 (Fig. 7D). This scenario does not apply to the V181A mutation because the V181A replicon is capable of replicating itself. Replication of the V181A replicon could occur in some cells before the wild-type p28 translated from a cointroduced wild-type replicon reaches the SIE-eliciting concentration threshold. Conversely, wild-type TCV could still replicate in a fraction of these cells because the V181A mutant p28 protein could only exert SIE inefficiently.

To summarize, the current study identifies two single-aminoacid mutations in TCV p28 that differentially impaired the two opposite functions of p28 (replication and SIE elicitation), hence decoupling these two functions of $\mathrm{p} 28$. These mutations will allow us to investigate the impact of SIE in the rate of mutation accumulation in TCV populations, providing insights regarding the evolutionary rationale for preserving SIE functionality in virus infections. These investigations would, in turn, unravel potential targets for antiviral therapy, and prompt novel management strategies for virus diseases.

\section{MATERIALS AND METHODS}

\section{Constructs.}

Constructs 2X35S::TCV_sg2R, p28-HA, p28 (tag-free), and Core35S::p88 (tag-free) were described in previous studies (Zhang et al. 2017; Zhang et al. 2019). The 2X35S::p28-GFP construct was a slightly modified version of the previous $\mathrm{p} 28$ GFP. The new version replaced the NotI site (GCGGCCGCA) linking p28 and GFP coding sequences with a BamHI site plus three additional nucleotides (GGATCCGGA). Nearly all deletion mutants, as well as single-amino-acid substitution mutants, were generated using synthetic gBlocks fragments (IDT, Coralville, IA, U.S.A.) that were integrated into various backbone plasmids (e.g., p28-GFP, p28-HA, or TCV_sg2R) using Gibson Assembly cloning (NEB, Ipswich, MA, U.S.A.). The identities of all new constructs were verified with Sanger sequencing. The constructs for the BIFC assay were assembled by fusing the NVen and CVen fusion tags to the $\mathrm{C}$ termini of $\mathrm{p} 28$, p28(V181A), and p28(F182A), using the 2X35S::p28-GFP construct backbone. The cDNA sequence of NVen and CVen was derived from the article of Gookin and Assmann (2014).

\section{Agroinfiltration.}

Upon verification, all of the constructs were introduced into Agrobacterium tumefaciens strain $\mathrm{C} 58 \mathrm{C} 1$ with electroporation (Qu et al. 2005, 2003). To carry out the experiments described in the Results section, various combinations of Agrobacterium suspensions were mixed together and delivered into $N$. benthamiana leaves as described (Qu et al. 2003; Zhang et al. 2015, 2017; Zhang et al. 2019). A p19-expressing Agrobacterium strain was included in all combinations to alleviate RNA silencing-mediated mRNA degradation.

\section{RNA extraction and Northern blotting.}

Total RNA was extracted from agroinfiltrated $N$. benthamiana leaves using the Direct-zol RNA Miniprep kit (Zymo Research, Irvine, CA, U.S.A.). To ensure consistency, four equivalent leaf sections derived from infiltrated leaves of four different plants were pooled before RNA extraction. The RNA extraction procedure included a DNase treatment step that removes DNA contamination. The RNA was then quantified with NanoDrop and subjected to Northern blotting as described $(\mathrm{Qu}$ et al. 2003; Zhang et al. 2015, 2017; Zhang et al. 2019).

\section{Protein extraction and Western blotting.}

Proteins were extracted from the agroinfiltrated $N$. benthamiana leaves following the protocol of Zhang et al. (2017). The polyclonal GFP and the monoclonal HA antibodies were purchased from Invitrogen. The AP-conjugated antirabbit (GFP) and antimouse (HA) secondary antibodies were purchased from Pierce and Novus, respectively. The HRP-conjugated antirabbit (GFP) secondary antibody was purchased from Abcam.

\section{Confocal microscopy and quantification of fluorescent cells.}

Confocal microscopic observations were carried out using a Leica Confocal microscope (TCS SP5) available at Molecular and Cellular Imaging Center at the Ohio Agricultural Research and Development Center, The Ohio State University. To quantify the percentage of cells expressing GFP, mCherry, or reconstituted mVenus (Figs. 5 and 7), the agroinfiltrated $N$. benthamiana leaf sections were photographed under the confocal microscope at low magnification $(\times 10)$ and for three separate channels (GFP/mVenus, mCherry, and merged). For each treatment, five independent leaf sections were photographed, resulting in 15 images. These images, typically encompassing 40 to 50 cells, were then divided into 100 equal subdivisions with the grid line feature of the PhotoShop software. Subdivisions that were more than $50 \%$ filled with a given fluorescence (GFP, mCherry, or both) were counted as positive for the respective protein, the total counts of which represent a percentage in a given image. For each treatment, five counts were obtained, and standard deviation were computed using these counts. In the case of BIFC experiments, the number of cells in each of the selected leaf sections was estimated using a coexpressed ER-mCherry protein (not shown). The reconstituted mVenus foci were counted for each of the five leaf sections, and the counts were quantified using box plots. 


\section{ACKNOWLEDGMENTS}

We thank the labs of L. Stewart and P. Redinbaugh for generously sharing equipment and other members of the Qu lab for stimulating discussions.

\section{LITERATURE CITED}

Andreu-Moreno, I., and Sanjuán, R. 2018. Collective infection of cells by viral aggregates promotes early viral proliferation and reveals a cellularlevel allee effect. Curr. Biol. 28:3212-3219.e4.

Bergua, M., Zwart, M. P., El-Mohtar, C., Shilts, T., Elena, S. F., and Folimonova, S. Y. 2014. A viral protein mediates superinfection exclusion at the whole-organism level but is not required for exclusion at the cellular level. J. Virol. 88:11327-11338.

Cao, M., Ye, X., Willie, K., Lin, J., Zhang, X., Redinbaugh, M. G., Simon, A. E., Morris, T. J., and Qu, F. 2010. The capsid protein of Turnip crinkle virus overcomes two separate defense barriers to facilitate systemic movement of the virus in Arabidopsis. J. Virol. 84:7793-7802.

Chen, Y.-H., Du, W., Hagemeijer, M. C., Takvorian, P. M., Pau, C., Cali, A., Brantner, C. A., Stempinski, E. S., Connelly, P. S., Ma, H.-C., Jiang, P., Wimmer, E., Altan-Bonnet, G., and Altan-Bonnet, N. 2015. Phosphatidylserine vesicles enable efficient en bloc transmission of enteroviruses. Cell 160:619-630.

Dietrich, C., and Maiss, E. 2003. Fluorescent labelling reveals spatial separation of potyvirus populations in mixed infected Nicotiana benthamiana plants. J. Gen. Virol. 84:2871-2876.

Folimonova, S. Y. 2012. Superinfection exclusion is an active virus-controlled function that requires a specific viral protein. J. Virol. 86:5554-5561.

Folimonova, S. Y. 2013. Developing an understanding of cross-protection by Citrus tristeza virus. Front. Microbiol. 4:76.

Gookin, T. E., and Assmann, S. M. 2014. Significant reduction of BiFC non-specific assembly facilitates in planta assessment of heterotrimeric G-protein interactors. Plant J. 80:553-567.

Julve, J. M., Gandía, A., Fernández-Del-Carmen, A., Sarrion-Perdigones, A., Castelijns, B., Granell, A., and Orzaez, D. 2013. A coat-independent superinfection exclusion rapidly imposed in Nicotiana benthamiana cells by tobacco mosaic virus is not prevented by depletion of the movement protein. Plant Mol. Biol. 81:553-564.

Kodama, Y., and Hu, C.-D. 2010. An improved bimolecular fluorescence complementation assay with a high signal-to-noise ratio. Biotechniques 49:793-805

May, J., Johnson, P., Saleem, H., and Simon, A. E. 2017. A sequenceindependent, unstructured internal ribosome entry site is responsible for internal expression of the coat protein of turnip crinkle virus. J. Virol. 91: e02421-16.

Mirabelli, C., and Wobus, C. E. 2018. All aboard! Enteric viruses travel together. Cell Host Microbe 24:183-185.

Miyashita, S., Ishibashi, K., Kishino, H., and Ishikawa, M. 2015. Viruses roll the dice: The stochastic behavior of viral genome molecules accelerates viral adaptation at the cell and tissue levels. PLoS Biol. 13:e1002094.

Miyashita, S., and Kishino, H. 2010. Estimation of the size of genetic bottlenecks in cell-to-cell movement of soil-borne wheat mosaic virus and the possible role of the bottlenecks in speeding up selection of variations in trans-acting genes or elements. J. Virol. 84:1828-1837.

Nethe, M., Berkhout, B., and van der Kuyl, A. C. 2005. Retroviral superinfection resistance. Retrovirology 2:52.

Powers, J. G., Sit, T. L., Qu, F., Morris, T. J., Kim, K.-H., and Lommel, S. A. 2008. A versatile assay for the identification of RNA silencing suppressors based on complementation of viral movement. Mol. Plant-Microbe Interact 21:879-890.

Qu, F., Ren, T., and Morris, T. J. 2003. The coat protein of turnip crinkle virus suppresses posttranscriptional gene silencing at an early initiation step. J. Virol. 77:511-522.
Qu, F., Ye, X., Hou, G., Sato, S., Clemente, T. E., and Morris, T. J. 2005. RDR6 has a broad-spectrum but temperature-dependent antiviral defense role in Nicotiana benthamiana. J. Virol. 79:15209-15217.

Qu, F., Ye, X., and Morris, T. J. 2008. Arabidopsis DRB4, AGO1, AGO7, and RDR6 participate in a DCL4-initiated antiviral RNA silencing pathway negatively regulated by DCL1. Proc. Natl. Acad. Sci. U.S.A 105:14732-14737.

Schaller, T., Appel, N., Koutsoudakis, G., Kallis, S., Lohmann, V. Pietschmann, T., and Bartenschlager, R. 2007. Analysis of hepatitis C virus superinfection exclusion by using novel fluorochrome gene-tagged viral genomes. J. Virol. 81:4591-4603.

Shaner, N. C., Steinbach, P. A., and Tsien, R. Y. 2005. A guide to choosing fluorescent proteins. Nat. Methods 2:905-909.

Simon, K. O., Cardamone, J. J., Jr., Whitaker-Dowling, P. A., Youngner, J. S., and Widnell, C. C. 1990. Cellular mechanisms in the superinfection exclusion of vesicular stomatitis virus. Virology 177:375-379.

Takahashi, T., Sugawara, T., Yamatsuta, T., Isogai, M., Natsuaki, T., and Yoshikawa, N. 2007. Analysis of the spatial distribution of identical and two distinct virus populations differently labeled with cyan and yellow fluorescent proteins in coinfected plants. Phytopathology 97: 1200-1206.

Tatineni, S., and French, R. 2016. The coat protein and NIa protease of two Potyviridae family members independently confer superinfection exclusion. J. Virol. 90:10886-10905.

Tscherne, D. M., Evans, M. J., von Hahn, T., Jones, C. T., Stamataki, Z., McKeating, J. A., Lindenbach, B. D., and Rice, C. M. 2007. Superinfection exclusion in cells infected with hepatitis $\mathrm{C}$ virus. J. Virol. 81:3693-3703.

White, K. A., Skuzeski, J. M., Li, W., Wei, N., and Morris, T. J. 1995. Immunodetection, expression strategy and complementation of turnip crinkle virus p28 and p88 replication components. Virology 211: 525-534.

Zhang, S., Sun, R., Guo, Q., Zhang, X.-F., and Qu, F. 2019. Repression of turnip crinkle virus replication by its replication protein $\mathrm{p} 88$. Virology 526:165-172.

Zhang, X., Zhang, X., Singh, J., Li, D., and Qu, F. 2012. Temperaturedependent survival of Turnip crinkle virus-infected Arabidopsis plants relies on an RNA silencing-based defense that requires dc12, AGO2, and HEN1. J. Virol. 86:6847-6854.

Zhang, X.-F., Guo, J., Zhang, X., Meulia, T., Paul, P., Madden, L. V., Li, D., and Qu, F. 2015. Random plant viral variants attain temporal advantages during systemic infections and in turn resist other variants of the same virus. Sci. Rep. 5:15346.

Zhang, X.-F., and Qu, F. 2016. Cross protection of plant viruses: Recent developments and mechanistic implications. Pages 241-250 in: Current Research Topics in Plant Virology. A. Wang and X. Zhou, eds. Springer International Publishing, Cham, Switzerland.

Zhang, X.-F., Sun, R., Guo, Q., Zhang, S., Meulia, T., Halfmann, R., Li, D., and $\mathrm{Qu}, \mathrm{F}$. 2017. A self-perpetuating repressive state of a viral replication protein blocks superinfection by the same virus. PLoS Pathog. 13 e1006253.

Zhang, X.-F., Zhang, S., Guo, Q., Sun, R., Wei, T., and Qu, F. 2018. A new mechanistic model for viral cross protection and superinfection exclusion. Front. Plant Sci. 9:40.

Zhou, X., Sun, K., Zhou, X., Jackson, A, O., and Li, Z. 2019. The matrix protein of a plant rhabdovirus mediates superinfection exclusion by inhibiting viral transcription. J. Virol. 93:e00680-19.

Ziebell, H., and Carr, J. P. 2010. Cross-protection: A century of mystery. Pages 211-264 in: Advances in Virus Research. Natural and Engineered Resistance to Plant Viruses, Part II. J. P. Carr and G Loebenstein, eds. Elsevier, London, U.K.

Zou, G., Zhang, B., Lim, P.-Y., Yuan, Z., Bernard, K. A., and Shi, P.-Y. 2009. Exclusion of West Nile virus superinfection through RNA replication. J. Virol. 83:11765-11776. 